\title{
VALIE EXPORT
}

Flux narratif versus matérialité du flux

Materialität des Ausflusses contra Erzählfluss: VALIE EXPORT

Material versus Narrative Flow: VALIE EXPORT

\section{Andréa LAUTERWEIN}

\section{OpenEdition}

\section{Journals}

Édition électronique

URL : http://journals.openedition.org/ceg/3815

DOI : $10.4000 /$ ceg.3815

ISSN : 2605-8359

\section{Éditeur}

Presses Universitaires de Provence

\section{Édition imprimée}

Date de publication : 15 novembre 2018

Pagination : 143-156

ISBN : 979-10-320-0183-7

ISSN : 0751-4239

Référence électronique

Andréa LAUTERWEIN, « VALIE EXPORT », Cahiers d'Études Germaniques [En ligne], 75 | 2018, mis en ligne le 25 avril 2020, consulté le 28 novembre 2020. URL : http://journals.openedition.org/ceg/3815 ; DOI : https://doi.org/10.4000/ceg.3815 


\title{
VALIE EXPORT
}

\section{Flux narratif versus matérialité du flux}

\author{
Andréa LAUTERWEIN \\ Université Sorbonne Nouvelle / CEREG (EA 4223)
}

En $1967^{1}$, VALIE EXPORT, artiste multimédia née en 1940 à Linz, montre les règles féminines comme on ne les avait jamais vues auparavant, dans toute leur réalité matérielle : assise sur un mur blanc, assez haut, elle urine alors qu'elle a ses règles. Trente-cinq ans plus tard, dans un entretien avec l'historienne de l'art Elisabeth Lebovici, VALIE EXPORT se souvient de cette action sans public qui fut filmée par sa sœur : "La pisse tachée courait le long du mur, créant un motif abstrait, rouge et liquide ${ }^{2}$." Menstruationsfilm, film muet en couleur de trois minutes, aujourd'hui porté disparu, est le début d'une œuvre iconique et réflexive autour du corps féminin et des distorsions médiatiques qui lui sont imposées.

De cette première action filmée, VALIE EXPORT retient aussitôt les dimensions plastique et critique. Entre 1967 et 1973, une période où l'artiste côtoie les actionnistes viennois ${ }^{3}$, la matière des règles féminines est mise en perspective dans quatre œuvres et quatre médias (action, performance, photographie, film) :

- Menstruationsfilm, une action filmée de 1967;

- Abstract film No. I, une performance expérimentale filmée de 1967/ 1968 (ill. 1);

- Menstruation, un cycle de photographies inachevé de 1973 (ill. 2);

- Mann \&t Frau Ct Animal, un film expérimental de 1973 (ill. 3).

Cette matérialisation surprenante des menstrues agit comme un choc, car elle contrarie un flux narratif ancestral destiné à occulter les règles féminines.

En vue de mieux comprendre certains aspects de la mémoire anthropologique qui sous-tend cette dématérialisation des menstrues, alors même qu'elles sont souvent considérées comme le principal marqueur de la différence sexuelle ${ }^{4}$,

1. La même année, l'artiste, née Waltraud Lehner et épouse Hollinger, se défait de ses patronymes pour "s'exporter" sous l'étiquette d'une marque de fabrique de cigarettes, VALIE EXPORT, témoignant à la fois de la réification de son corps et de son "expertise" en la matière.

2. Elisabeth Lebovici, "Entretien avec Valie Export », in Valie Export, cat. exp. Montreuil, Éditions de l'œil, 2003, p. 145.

3. Voir les reproductions des œuvres sur [http://www.valieexport.at/en/werke], dernière consultation le 24 septembre 2017.

4. Cf. Iris Marion Young, " Menstrual Meditations ", in On Female Body Experience: Throwing like $a$ Girl and Other Essays, London, Oxford University Press, 2005, p. 71. L'anthropologue Alain 
nous évoquerons d'abord les grandes narrations ainsi que les discours scientifiques qui consolident le tabou. Nous reviendrons ensuite sur le contexte artistique spécifique dans lequel ont émergé les œuvres de VALIE EXPORT portant sur la menstruation pour proposer une interprétation du rôle de la matérialité dans les travaux cités ci-dessus.

\section{Des récits qui dématérialisent}

Les menstrues constituent un phénomène corporel spécifiquement féminin. Entre la puberté et la ménopause, durant cinq à sept jours, à intervalles plus ou moins réguliers, un flux s'écoule de l'entre-jambe des femmes. Les grands récits qui cherchent à expliquer l'origine et la vocation de ce phénomène entouré de mystère furent énoncés la plupart du temps dans une perspective androcentrique et contribuèrent à confiner les femmes aux marges de la société. La théologie, la philosophie, la médecine, les disciplines historiques, la psychanalyse poursuivent le fil de ces narrations avec des discours prétendument scientifiques en consolidant le tabou ${ }^{5}$. La littérature critique identifie aujourd'hui plusieurs causes de ce tabou conduisant à dématérialiser le phénomène : de la nécessité pratique d'une vie dans des conditions dites "primitives" à une compensation de l'angoisse de castration en passant par le contrôle des naissances et la stabilisation de structures sociales dualistes ${ }^{6}$.

L'argument le plus résistant dans les récits et les discours qui dématérialisent les menstrues est celui de l'impureté, entraînant le dégoût du sang menstruel. Dans les religions sémitiques, les femmes sont considérées en état d'impureté rituelle pendant leurs règles et sont exclues d'un certain nombre de pratiques sociales ${ }^{7}$. Ces superstitions charrient une série de croyances transmises de génération en génération jusqu'à présenter le sang menstruel comme un agent de contagion infectieuse. La terreur inspirée par le sang qui s'écoule, et le non-

Testart situe le tabou des règles à l'origine de la division du travail et de la constitution du genre : l'interdiction symbolique de mélanger les sangs (sang menstruel et jaillissant) ferait que les femmes travaillent les matières tendres, molles et flexibles alors que les hommes travaillent les matières dures. Alain Testart, L’Amazone et la Cuisinière. Anthropologie de la division sexuelle du travail, Paris, Gallimard, 2014.

5. À la fois au sens anthropologique d'une « chose qu'il n'est pas permis de toucher parce qu'il (elle) est investi(e) momentanément ou non d'une puissance sacrée jugée dangereuse ou impure " et au sens plus général d'un " interdit d'ordre culturel et/ ou religieux qui pèse sur le comportement, le langage, les mœurs ", [http://www.cnrtl.fr/definition/tabou], dernière consultation le 24 septembre 2017.

6. Voir notamment Victoria Louise Newton, Everyday Discourses of Menstruation : Cultural and Social Perspectives, London, Palgrave Macmillan, 2016; Glenda Lewin Hufnagel, A History of Women's Menstruation from Ancient Grece to the Twenty-first Century : Psychological, Social, Medical, Religious and Educational Issues, Lewiston NY, Mellen Press, 2012 ; Kristina Hohage, Menstruation. Eine explorative Studie zur Geschichte und Bedeutung eines Tabus, Hamburg, Verlag Dr. Kovac, 1998; Françoise Edmonde Morin, La rouge différence ou les rythmes de la femme, Paris, Seuil, 1985.

7. À la source, le texte du Lévitique qui contient les injonctions d'une loi scellant pour des siècles la notion d'impureté (15, 9-25). 
respect des restrictions entraînent ainsi des sanctions qui peuvent aller jusqu'à l'exclusion du groupe. Parfois les restrictions appliquées à l'origine durant la période des règles sont étendues à toutes sortes d'occasions, probablement en raison de l'imprévisibilité du cycle féminin, devenant ainsi un instrument d'oppression des femmes ${ }^{8}$.

Ce lot de superstitions a des conséquences graves pour la vie des femmes. Il n'est pas le seul apanage des sociétés dites "primitives" : d'Hippocrate à nos jours, les croyances traditionnelles s'appuient fréquemment sur un discours médical qui leur donne un fondement pseudo-scientifique : les menstrues sont décrites comme des "purges naturelles " constituées de substances toxiques capables de faire se faner les fleurs et les arbres, de faire tourner le lait et d'avarier la viande. Dans ce domaine, la théorie s'émancipe difficilement des constructions mythologiques et se fonde volontiers sur des narrations : la fameuse théorie médicale des "ménotoxines " élaborée en 1920 à Vienne par le docteur Bela Schick, démentie vers la fin des années 1940 mais restée profondément ancrée dans les esprits ${ }^{9}$, se base sur le récit suivant :

Ayant un jour offert un magnifique bouquet de roses à une jeune fille de sa connaissance, le docteur Schick avait eu la surprise de constater que, dès le lendemain, les roses étaient fanées; or la jeune fille avait ses règles, et avait eu elle-même plusieurs fois l'occasion de constater le phénomène lors d'occasions précédentes ${ }^{10}$.

À partir de cette observation romancée, Schick pose le principe de l'existence des " ménotoxines ", substances nocives éliminées par la peau de la femme indisposée qui seraient responsables de différents phénomènes de pourrissement et de fanaison. Mais le préjugé d'impureté, manifestement lié au mystère de l'écoulement et à la mémoire d'un échec reproductif, ne s'arrête pas à des questions d'hygiène et de fertilité, et peut aller jusqu'à attribuer des pouvoirs maléfiques à la femme réglée. L'association fantasmatique de la femme réglée avec le vampire a pu conduire à sa criminalisation au XIX ${ }^{\mathrm{e}}$ siècle lorsque de nombreux médecins reliaient la criminalité féminine à la supposée labilité morale durant ses règles ${ }^{11}$.

8. On peut citer l'exemple très marquant des îles Marquises : chez les Marquisiens, il est interdit aux femmes de passer au-dessus de tout objet ou structure durant leurs règles et, par extension, de s'asseoir sur la selle d'un homme en temps normal, d'aller en canoë, de s'asseoir sur une chaise ou sur le porche d'une maison en présence d'un enfant. En cas de non-respect de la règle, l'objet et/ ou la femme sont passibles de destruction. Cf. Robert Carl Suggs, Marquesan Sexual Behavior, Harcourt, Brace \& World, 1966, p. 28-35.

9. L'historien de la médecine E. Püschel explique que dans certaines régions d'Autriche, les femmes indisposées sont écartées de la conservation des aliments comme des bourgeons et des arbres en fleurs. Erich Püschel, Die Menstruation und ihre Tabus. Ethnologie und kulturelle Bedeutung. eine ethno-medizingeschichtliche Übersicht, Stuttgart/ New York, Schattauer, 1988, p. 29-30. Voir aussi, pour la France, Yvonne Verdier, Façons de dire, façons de faire, Paris, Gallimard, 1979.

10. Cf. Jean-Yves Naour, Catherine Valenti, " Du sang des femmes. Histoire médicale de la menstruation à la Belle Epoque ", Clio. Histoire, femmes et sociétés 14, 2001, [http://clio.revues. org/114], dernière consultation le 24 septembre 2017.

11. Ibid. 
Le discours psychanalytique est lui aussi à l'origine d'une série de narrations négatives. Freud, dans Totem et Tabou, explique le tabou des règles féminines à partir de la théorie androcentrique de l'angoisse de castration; les femmes relieraient inconsciemment le sang menstruel à la défloration et l'associeraient à une blessure physique. À sa suite, Hélène Deutsch interprétera également la menstruation comme une expérience inquiétante pour la jeune fille qui la découvre, si bien qu'elle percevrait le sang menstruel comme une castration symbolique. Pour le psychanalyste Erik Erikson les menstrues demeureraient le symbole négatif de la " perte d'un enfant ", tandis que Bettelheim ou Devereux expliquent la dévalorisation des règles par l'envie masculine de la faculté de procréer passivement ${ }^{12}$.

La théorie féministe a longtemps entretenu cette terreur de la biologie féminine, qui semble avoir été intériorisée par les femmes. Simone de Beauvoir, dans Le Deuxième Sexe, exprimait le point de vue dominant d'une génération de féministes honteuses de leur corporéité ${ }^{13}$ :

C'est alors que chez la fillette naît ou s'exagère le dégoût de son corps trop charnel. Et passé la première surprise, le désagrément mensuel ne s'efface pas pour autant : chaque fois, la jeune fille retrouve le même dégoût devant cette odeur fade et croupie qui monte d'elle-même - odeur de marécage, de violettes fanées - devant ce sang moins rouge que celui qui s'échappait de ses écorchures enfantines ${ }^{14}$.

La redécouverte des mythes féminins et des rites matriarcaux durant les années 1970 introduira un récit positif de la menstruation, au point de faire apparaître la malédiction comme une bénédiction qui permettrait aux femmes de vivre plus intensément leur corps et leur sexualité ${ }^{15}$. À partir des années 1990, la théorie du genre butera sur la résistance des corps biologiques, et en particulier celle de la menstruation, qui influe fortement sur l'autoreprésentation et la temporalité du corps vécu ${ }^{16}$. En tant que signe matériel ultime d'un genre qui interroge ses limites, les menstrues sont aujourd'hui au cœur des controverses féministes. Contre leur invisibilité sociale, elles sont parfois même revendiquées comme l'ultime indice de différenciation des genres ${ }^{17}$.

12. Voir pour ce passage Hohage, Menstruation, p. 253-259.

13. Cf. Sylvie Chaperon, "La réception du Deuxième Sexe en Europe ", in Encyclopédie pour une histoire nouvelle de l'Europe, 2016, [http://www.ehne.fr/node/951], dernière consultation le 24 septembre 2017.

14. Simone de Beauvoir, Le Deuxième Sexe, vol II, Paris, Gallimard (coll. Poches), 1976, p. 70.

15. Voir Penelope Shuttle, Peter Redgrove, The Wise Wound : Menstruation and Everywoman, Londres, Gollanzc, 1978. Traduit en allemand en 1980 et réédité plusieurs fois chez Fischer sous le titre Die weise Wunde Menstruation.

16. Voir Iris Marion Young, "Menstrual Meditations », p. 97-122 ou Kathleen Lennon, " Feminist Perspectives on the Body ", The Stanford Encyclopedia of Philosophy (Fall 2014 Edition), [https://plato.stanford.edu/archives/fall2014/entries/feminist-body/], dernière consultation le 24 septembre 2017.

17. L'exception de la publicité pour le marché de l'hygiène intime est trompeuse, car elle contribue à occulter l'existence des menstrues en encourageant les femmes à les faire disparaître. Il est suggéré que les femmes sont d'une part victimes de leur biologie, et d'autre part que l'occultation de cette malédiction leur permet de s'en libérer. Comme dans les jeux de vidéo de guerre destinés aux enfants, où le sang des victimes est bleu pour masquer la pulsion de mort, le sang est 


\title{
Sang pour sang : I'exception féminine parmi les actionnistes viennois
}

L'histoire de l'art, et tout particulièrement la peinture chrétienne, dégouline de sang et de tortures ; le sang animal est utilisé comme pigment depuis la préhistoire, mais il est plus rare de trouver des peintures avec du sang humain. Au XX siècle, on associe immédiatement le sang aux pratiques radicales de la mouvance des actionnistes viennois à laquelle les performances de VALIE EXPORT sont souvent associées, mais dans laquelle elle occupe assurément une place à part ${ }^{18}$.

Comme d'autres mouvances artistiques des années 1960 et 1970, l'actionnisme autrichien cherche à se libérer des assignations anciennes du corps en engageant librement le corps comme objet et matériau :

\begin{abstract}
Die Regression aufs Material als allgemeines Prinzip der Entwicklung der bildenden Kunst in den letzten Dekaden ist Anzeichen für eine Methode der Wahrnehmung, die durch einen Körper nach einem anderen Körper zielt, die sich in der Welt ereignet und nicht im falschen Schein der schönen Künste. Im Horizont des Leibes und der Welt angesiedelt, ist das künstlerische Medium der Körper. Der menschliche Körper selbst ist das Kunstwerk, das Material ${ }^{19}$.
\end{abstract}

Dans le contexte de la société chrétienne et postfasciste autrichienne, l'actionnisme critique tout particulièrement la répression de la pulsion de mort, considérée comme hypocrite, à travers un traitement identique de l'objet, du corps humain et du matériau. L'actualisation des pulsions agressives par le sacrifice ritualisé de corps animaux, leur mise en pièce, le montage de leurs entrailles ou la mise en scène lors d'actions collectives impliquant les artistes eux-mêmes et/ ou des "acteurs passifs" de préférence des femmes, sont présentés comme une alternative pré-chrétienne et dionysiaque à vocation cathartique pour l'équilibre psychique ${ }^{20}$. Or l'actionnisme viennois est presque entièrement porté par des hommes, dont certains sont marqués par l'expérience de brutalisation de la guerre ou du front, notamment Otto Mühl avec ses "Materialaktionen " où l'identification d'une "violence structurelle " donne toute licence à une "violence non-structurée ${ }^{21}$ ". Günter Brus et Roland Schwarzkogler, venus de la peinture informelle, mais s'inscrivant dans la droite lignée des peintres

dématérialisé et présenté comme un fluide bleu ciel ou rose clair. Les femmes intègrent les valeurs de la discrétion, de la honte, du secret et sont culpabilisées.

18. Le livre de documentation coédité à chaud avec Peter Weibel en 1970, dans lequel on trouve les actions de VALIE EXPORT réalisées avant cette date, donne une définition assez large de ce " groupe ", qui sera relativisée plus tard. Cf. Valie Export, Peter Weibel (éd.), Bildkompendium Wiener Aktionismus und Film, Frankfurt a. M., Kohlkunstverlag, 1970.

19. Peter Weibel, Kritik der Kunst/ Kunst der Kritik, Wien, Jugend Ct Volk, 1973, s.p., cité dans Valie Export, "Feministischer Aktionismus. Aspekte " in Gislind Nabakowski, Helke Sander, Peter Gorsen (dir.), Frauen in der Kunst, vol. 1, Frankfurt a. M., Suhrkamp, 1980, p. 139.

20. Thomas Dreher, Performance Art nach 1945. Aktionstheater und Intermedia, München, Wilhelm Fink, 2001, p. 201-204.

21. Wolfgang Kemp, Der explizite Betrachter. Zur Rezeption zeitgenössischer Kunst, Konstanz, Konstanz University Press, 2015, p. 58-59. 
expressionnistes et leurs représentations du corps torturé et souffrant, décrivent l'action comme une abréaction physique visant à retourner l'intérieur vers l'extérieur, afın de libérer des forces inconscientes réprimées. S’il arrive que les membres de l'actionnisme viennois interrogent aussi de manière provocatrice les assignations traditionnelles du genre en engageant le corps ${ }^{22}$, les corps féminins, toujours dénudés, n’y ont jamais d'autre fonction que celle d'un objet singulièrement dégradé.

L'actionnisme viennois véhicule en effet une image du féminin passive et fantasmatique, bien éloignée des aspirations de VALIE EXPORT. Dans l'essai "Actionnisme féministe " de 1977, elle différenciera sa propre pratique du corps comme matériau en présentant le "drame du matériau " comme un " drame de significations ", et la " pensée matériau " comme une " libération du caractère objectal de la femme " :

Egal ob Körper oder Dinge das Material waren, man sieht das Materialdrama war eigentlich ein Bedeutungsdrama. Das Material als Bühne verschiedener Bedeutungen verarbeitete und integrierte nicht nur die Erlebnisse von Menschen, sondern aktivierte auch ihre Erlebnisfähigkeit, schärfte das Bewusstsein für die durch das Material entfalteten Bedeutungen [...]. So wie das "Materialdenken die Produkte des Menschen von ihrem Dingcharakter befreien soll ", könnte man paraphrasieren, so soll es im Feministischen Aktionismus die Produkte des Mannes, nämlich die "Frauen", von ihrem Dingcharakter befreien - Materialdenken als Befreiung der Frau vom Dingcharakter. [...] Kunstgeschichtlich hat der Feministische Aktionismus die gleichen Quellen wie der Aktionismus; doch einige andere, neue kommen dazu. [...] Die wichtigste Quelle ist allerdings die Geschichte der weiblichen Erfahrung. [...] Die Blutspur, die uns allen mit unsichtbarer Tinte eingeschrieben ist, als gattungsgeschichtliches Tabu, ist das Material des feministischen Aktionismus. Und der Materialien gibt es da genug, ja ungeheuer viele. ${ }^{23}$

Cette différence spécifique qui se cristallise dans " l'histoire de l'expérience féminine " se révèle tout particulièrement dans l'utilisation faite du matériau du sang menstruel. Chez Hermann Nitsch, par exemple, l'ouverture symbolique des corps et la monstration des sécrétions corporelles avaient pour objectif de matérialiser des abstractions mythiques, en particulier celles des récits religieux et de leur imagerie sanguinolante de la Passion. En 1966, il crée l'action "Erste heilige Kommunion " dans le cadre des Abreaktions-Spiele à la Galerie Dvorak à Vienne. Il y déverse des hectolitres d'hémoglobine d'origine animale en les associant à un crucifix et à une serviette périodique maculée de sang menstruel ${ }^{24}$. Cette action, où le matériau des sèmes menstruels est utilisé de la même manière que les matières fécales ou les entrailles d'animaux, perpétue la symbolique de l'horreur des entrailles maternelles, de la souillure et de la dégradation. Nitsch révélait un tabou, à en croire du moins ses démêlés avec la police, mais la monstration du sang menstruel visait en premier lieu à

22. Jacques Lajarrige, "Mauvais genres : Les actionnistes viennois et le corps ", in Patrick Farges, Cécile Chamayou-Kuhn, Perin Emel Yavuz (dir.), Le lieu du genre. La narration comme espace performatif du genre, Paris, Presses de la Sorbonne Nouvelle, 2011, p. 21-37.

23. Valie Export, "Feministischer Aktionismus ", p. 140-142.

24. Danielle Spera, Hermann Nitsch : Leben und Arbeit, Wien, Brandstätter, 2005, p. 95. 
blasphémer le récit de la séparation du sang impur et du sang sacré ${ }^{25}$. L'emploi de serviettes périodiques imbibées de sang pour 'soigner' le membre viril du modèle dans une série d'actions intitulées Penisbespülungen (dès 1965), ou les bandages de Schwarzkogler qui s'en inspirèrent, sont peut-être des jeux avec l'ambiguïté genrée ${ }^{26}$, il n'en demeure pas moins que ce jeu avec les attributs féminins et les écoulements périodiques est associé soit à l'idée du vagin comme plaie ouverte choquante qu'il s'agirait de soigner et d'occulter, soit au mythe de la vagina dentata qui renvoie lui-même à l'angoisse de castration.

Il s'agit donc bien de récits anxiogènes, qui occultent l'expérience subjective féminine que VALIE EXPORT cherche à matérialiser en toute sobriété dans ses travaux sur la menstruation. Chez elle, la mise en scène de la matière menstruelle ne se justifie par aucun rituel liturgique ou chamanique; aussi cherchera-t-on vainement une trace de mysticisme ou de pathos blasphématoire. Par ailleurs, la manipulation de modèles humains passifs comme matériau lui est étrangère. C'est son propre corps qu'elle met en danger, et elle ne montre que rarement des blessures ouvertes, tout juste des incisions modérées qui visent métaphoriquement le domaine du subcutané ${ }^{27}$. La " coupure, la déformation, le sang "dans ses actions ne se présentent pas comme " le reflet de besoins sado-masochistes ", ni comme "les tensions de pulsions abnormes ", mais comme des concepts rivés aux corps des femmes, comme les stigmates corporels de la domination historique ${ }^{28}$. La matérialité physique du corps et ses prolongements médiatiques visent l'objectivation critique d'états psychiques et questionnent leur origine socio-politique. Ainsi, dans un poème de 1973 intitulé " Matériau de la réalité " [Material der Wirklichkeit], VALIE EXPORT décrit bien la nécessité de montrer la violence historique inscrite dans le corps des femmes comme un acte « dépourvu de nerfs " :

MATERIAL DER WIRKLICHKEIT, eine frage, // die vergangenheit in staubige ritzen blasen /und ohne nerv/den hammer in die wunde schlagen //das blut suchen, lasst den fleischschmerz erfahren. / jänner $1973^{29}$.

25. On notera que les commentateurs se plaisent à écrire que ce sang menstruel est " faux ". L'artiste fut condamné à six mois de prison, car, comme le retient le protocole de police, " ce type de choses sont brûlées ou mises à la poubelle, même par les femmes les plus primitives, en dehors de l'espace public ". Cité par Monika Wagner, Das Material der Kunst : Eine andere Geschichte der Moderne, München, C.H. Beck, 2001, p. 226.

26. Lajarrige, "Mauvais genres ", p. 27-28.

27. Voir notamment l'action Remote, remote (1973) où l'artiste se coupe les cuticules avec un cutter dans une mise en scène complexe. Cf. Andrea Zell, Valie Export. Inszenierung von Schmerz : Selbstverletzung in den frühen Aktionen, Berlin, Verlag Dietrich Reimer, 2000, p. 86-108.

28. Valie Export, "Feministischer Aktionismus », p. 143 : "Wenn schon pathologische Momente wie Selbsthass, minderes Selbstbewusstsein, Identifikation mit Leid, Unterwerfung oder gar mit dem Unterdrücker aus solchen Aktionen interpretatorisch rezipiert werden, dann sind dies Momente der Wahrheit der Geschichte der Frau ".

29. Poème accompagnant la série de dessins Die Träume eines Kindes (1971-72) in VALIE EXPORT, splitter, Band I : Konfigurationen. Fotografien 1968-77, Linz, Edition Neue Texte, 1980, p. 94. 
Considérant son art et l'actionnisme féministe comme " une force historique objective $^{30}$ ", VALIE EXPORT serait donc plutôt à placer dans la filiation des artistes femmes du surréalisme (Meret Oppenheim, Dora Maar, Sophie Täuber-Arp) qui, au travers de leur " fétichisme surréel du matériau" ${ }^{31}$ ", montrent les "insignes d'une énigmatique mutilée ${ }^{32}$ " dont les significations révolutionnaires fournissent une " châne d'associations dans le drame du matériau actionniste ${ }^{33}$ " et, plus récemment, dans le sillage des femmes artistes américaines du Body Art (Gina Pane, Marina Abramović, Carolee Schneemann), qui revendiquent l'expérience intime du corps féminin et le dégagent des mythes et des projections masculines par le dénudement ou l'incision, se glissant ainsi sous une peau devenue surface de projection. Le désir de matérialiser les règles féminines y apparait également dans une logique d'auto-affirmation intimiste qui s'inscrit en faux contre le récit dominant ${ }^{34}$.

\section{La " pensée matériau » au contact du corps féminin et de ses flux}

VALIE EXPORT rompt donc radicalement avec une tradition artistique autrichienne où le genre féminin existe avant tout comme un fantasme. Dans le cadre de sa " pensée matériau " [Materialdenken], elle se sert de son propre corps, de son fonctionnement et de ses sensations, comme matériau objectif et producteur de sens. Or, comme le souligne Sabine Wilke, " la crainte d'une déformation de l'intégrité féminine par le langage verbal masculin est étendue à une analyse de la déformation du langage corporel des femmes, tel qu'il apparaît notamment dans les tableaux peints par des hommes ${ }^{35}$ " :

Wir haben bereits als eine Quelle des Feministischen Aktionismus die Auflehnung gegen die männliche Verbalsprache zitiert [...]. Doch auch in der Körpersprache war es nicht leicht, sein Wort zu finden, denn auch sie war überwiegend von Männerphantasien okkupiert ${ }^{36}$.

VALIE EXPORT a donc documenté ces déformations du langage corporel dans de nombreuses actions artistiques du début des années 1970. Les postures idéales du corps féminin, qui figent les narrations sur la corporéité féminine, y sont soumises à une étude ironique, notamment dans la série Körperstellungen :

30. Valie Export, "Feministischer Aktionismus », p. 144.

31. Ibid., p. 145.

32. Ibid., p. 141.

33. Ibid., p. 145.

34. Une semaine de mon sang menstruel (1973) de Gina Pane notamment, un dispositif faisant partie de l'action Autoportraits présentée à la Galerie Stadler à Paris en 1973 : sur sept cotons imbibés de son sang menstruel utilisés pendant une semaine précédant l'action puis disposés en ligne, l'artiste exposait la matérialité de sa " période ". [http://www.centrepompidou.fr/cpv/resource/ cLjMqo/rrbKX5d], dernière consultation le 24 septembre 2017.

35. Sabine Wilke, Dialektik und Geschichte : feministische Schreibpraxis in der Gegenwartsliteratur, Tübingen, Stauffenberg, 1995, p. 78.

36. Valie Export, "Feministischer Aktionismus », p. 160-161. 
Nachstellungen, où elle déconstruit un choix de stéréotypes de l'histoire de la peinture ${ }^{37}$. Les moyens utilisés dans cette entreprise de subversion critique des codes sont presque exclusivement la photographie et la vidéo que VALIE EXPORT comprend comme des extensions “objectivantes" de sa corporéité. Les média du film et tout particulièrement l'image électronique de la vidéo naissante ont en effet fait leur entrée dans le domaine de l'art contemporain dans un contexte militant où les artistes cherchent de nouveaux métalangages par l'investigation du corps : " Leurs usages artistiques ne sont pas encore codifiés qu'ils sont immédiatement appropriés par des femmes et reversés dans le champ de leur $\operatorname{art}^{38} »$. Dans un manifeste de 1973, VALIE EXPORT appelle ces nouveaux médias des " cubes de construction média " [mediale bausteine] :

gebt den frauen das wort, damit sie zu sich kommen können, wir frauen müssen, um zu einem von uns selbst bestimmten bild der frau kommen zu können und damit zu einer veränderten abbildung in der gesellschaftlichen funktion der frau, an der konstruktion der wirklichkeit via den medialen bausteinen teilhaben ${ }^{39}$.

Ces " cubes de construction média " lui permettront de déconstruire les liens de causalité des grands récits et d'explorer de nouveaux codes fondés sur l'expérience vécue, tout en promouvant la dimension plastique, matérielle et tactile des sensations corporelles, qui échappent dans une certaine mesure à la logique dramaturgique du récit dominant.

En se référant aux pionnières de l'avant-garde, VALIE EXPORT ira jusqu'à défınir l'art cinématographique [Film], qu'elle distingue du cinéma [Kino], comme l'art féminin par excellence car ses langages et ses codes, contrairement à ce qui se passe dans les arts plus anciens, ne seraient pas encore surdéterminés ${ }^{40}$. Avec son concept du " expanded cinema ", VALIE EXPORT remettra en cause le dispositif habituel du cinéma (où les spectateurs sont passifs, assis dans la salle obscure à regarder la projection d'images sur un écran selon un ordre narratif imposé) en élargissant le champ du filmique à la performance qui multiplie les points de vue ${ }^{41}$.

37. Dans les photomontages Die Geburtenmadonna ou Die Strickmadonna de 1976, elle subvertit la narration de la Vierge Marie par une Vierge moderne entourée de son appareillage électroménager; dans Nonpareille de 1976, elle pose en reproduisant les contorsions des corps féminins nus du Printemps de Botticelli, tout en étant habillée de vêtements contemporains, et en dénonce le ridicule. Cf. Valie Export, Körpersplitter, p.77-90.

38. Catherine Gonnard, Elisabeth Lebovici (dir.), Femmes artistes/ artistes femmes. Paris, de $1880 \dot{a}$ nos jours, Paris, Hazan, 2007, p. 312.

39. Valie Export, "Woman's art. A manifesto ", Neues Forum 228, 1973, p. 47, cité par Wilke, Dialektik und Geschichte, p. 76.

40. «Frauen waren es, die massgebend am Beginn und Aufbau der Filmgeschichte [...] mitgearbeitet haben. [...] Film wird den Frauen zum Medium der Selbstrepräsentation im Gegensatz zur Praxis des herrschenden Kinos ". Valie Export in Kunst mit Eigen-Sinn. Aktuelle Kunst von Frauen. Texte und Dokumentation, Wien, Löcker, 1985, p. 199.

41. Ainsi, dans la performance Tapp-und Tastkino répétée à plusieurs reprises entre 1968 et 1971 dans les rues de diverses villes européennes, VALIE EXPORT invente un " cinéma du toucher et de la palpation ", en déconnectant le cinéma de la hiérarchie de la pulsion scopique pour le reconnecter au toucher : habillée comme un homme-sandwich d'un petit cinéma en carton, elle invitait le spectateur à introduire ses mains à travers le rideau du " cinéma " (dans la limite de 33 
La performance filmée Abstract film No. I (1967/ 68) peut être considérée comme un premier refus très formel de la linéarité et des hiérarchies du récit, qui est ici remplacé par le flux et la sensualité brute des matériaux, divulgués par un dispositif complexe (ill. 1) : un miroir sur lequel un projecteur de cinéma envoie de la lumière est placé devant un écran réfléchissant; VALIE EXPORT verse sur ce miroir des liquides de différentes couleurs et viscosités, dont notamment du sang menstruel, et des images colorées et mouvantes apparaissaient sur l'écran. A priori il n'y a rien dans cette œuvre abstraite qui permette de faire le lien avec le vécu de l'artiste, et pourtant elle résulte de son expérience et de sa réflexion sur la corporéité féminine : le motif surdéterminé du miroir renvoie à la fois à l'autoréflexion du sujet et à une symbolique féminine ambivalente que VALIE EXPORT situe entre les rêves brisés et la " collecte des fragments d'une âme torturée ${ }^{42}$ ". Pris dans ce dispositif complexe de reflets, les matériaux actualisés par lesdits " cubes de construction média " cassent la linéarité de l'action et par conséquent brisent la logique dramaturgique du récit :

Ich möchte keine Geschichten erzählen, sondern was kann ich mit dem Material machen, wie kann ich Zeit einsetzen, wie kann ich in der Zeit zurück gehen oder nach vorne gehen, wie kann ich verschiedene psychische Zustände direkt miteinander konfrontieren ... wo auch eine Weiterführung geschieht, eben durch die Destrukturierung der Geschichte, durch einen Ablauf, durch formalen Einsatz, man muss Film im Film machen. Man kann Dia, Fotografie als dramaturgische Mittel im Film einsetzen ${ }^{43}$.

La structure associative de l'œuvre de VALIE EXPORT correspond au refus de la détermination historique du féminin par le récit ${ }^{44}$ et conduit à l'élaboration d'un langage filmique fait d' " images flottantes " [floating images] auxquelles est attribuée la faculté de produire de nouveaux codes ${ }^{45}$. Dans Abstract film $n^{0} 1$ (ill. 1), la présentation de signes non figuratifs et non codifiés, avec des matériaux concrets, dont on perçoit la mise en œuvre manuelle et la qualité matérielle, souligne aussi leur qualité haptique. Les images haptiques provoquent une perception qui n'est donnée normalement que par le sens du toucher, ce que Gilles Deleuze appelait la "vision haptique ", dépassant la dualité du tactile et de l'optique ${ }^{46}$. Ici, la matière du sang menstruel est montrée dans sa pure matérialité ;

secondes) et à toucher ses seins dénudés. Le " voyeur " peut donc toucher, mais au vu de tous, et se défait par là-même de son statut de voyeur; en offrant matériellement à saisir la promesse du corps féminin véhiculée par les médias, l'autoreprésentation féminine est corrigée, la perspective masculine déconstruite par le contact.

42. Valie Export, " Feministischer Aktionismus " (Die Klinik der zwei Spiegel), p. 156.

43. Valie Export, in Fotografie 12/1, 1984-5, p. 28.

44. VALIE EXPORT qualifie les performances de l'artiste américaine Carolee Schneemann dont elle se sent très proche comme un " théâtre du mouvement du corps dans l'espace qui est nonlittéraire, non-verbal [...] qui explore et explique l'empire féminin et ses signes voilés [...] ". Cf. Valie Export, "Feminismus \& Kunst ", Neues Forum 229, 1973, p. 60, cité par Wilke, Dialektik und Geschichte, p. 77.

45. Cf. Sigrid Eiblmayr, Gertrud Koch, Marlene Steeruwitz (dir.), Valie Export : Mediale Anagramme, Berlin, NGBK, 2017, p. 123 et Valie Export dans Sandra Frieden, Ricky McCormick et al. (dir.), Gender Perspectives in German Cinema, Boston, Berg, 1992, p. 174.

46. Gilles Deleuze, Logique de la sensation I, Paris, La Différence, 1996, p. 99-103. 
non comme un déchet immobilisé par la serviette hygiénique, servant à figer et à occulter le flux, mais en tant que flux dynamique et haptique.

Dans la performance filmée Menstruationsfilm (1967) que nous avons évoquée plus haut, la présentation matérielle de l'écoulement involontaire est doublée d'une expulsion active, comme le souligne Émilie Bouvard : " Le trouble transgressif est triple : sang menstruel et urine sont des matières réputées ignobles, le fait d'uriner procure un soulagement sinon un plaisir, celui de l'expulsion, lui-même tabou, et enfın, la femme expulse ici ces matières de façon active ${ }^{47}$ ". En déjouant le système de signes de la dématérialisation historique, qui condamne les femmes aux restrictions et à la passivité, cette présentation non-narrative des matériaux issus du corps féminin se dégage aussi des récits historiques qui associent le sang à une rhétorique de la souffrance et des immondices.

Dans sa continuité, le cycle de photographies Menstruation (1973) présente le résultat brut d'une semblable expulsion : il s'agit en effet de la vue plongeante sur une cuvette de WC à l'intérieur de laquelle flotte du sang menstruel clair autour d'un caillot plus foncé, précisant la dramaturgie de l'ensemble (ill. 2). Le contenu de la cuvette est disposé à la manière d'un œuf cassé que l'on associe spontanément à un échec de la procréation, mais qui, par l'objectivation crue de sa matérialité et sa mise en scène provocatrice, se transforme en image maîtrisée du "refus" de la procréation.

Le film expérimental Mann \&t Frau \&t Animal (1970/1973) associe les règles féminines à la jouissance féminine. Il commence avec une scène d'autoérotisme filmée en noir et blanc. La caméra s'attarde sur les détails de la salle de bain où cela se passe, notamment sur la mécanique de la plomberie, filmée de près dans toute sa matérialité métallique : robinets aux attributs phalliques, bonde aspirant vertigineusement l'eau mousseuse. "L'homme \& la femme " du titre sont là ; l'aspect " animal " peut être interprété avec Martine Beugnet comme " la matérialisation d'un devenir qui se soustrait aux fonctions phallocentriques de l'identité ${ }^{48}$ ". Avant de basculer dans l'immanence pure d'un dernier plan entièrement blanc qui échappe au monde des représentations ${ }^{49}$, l'avant-dernière séquence du film passe à la couleur, comme pour mieux signaler la " rouge différence " (ill. 3). On y voit l'image rapprochée d'un sexe féminin, à la manière de L'Origine $d u$ monde de Gustave Courbet, mais en plus réaliste, coloré par $\mathrm{du}$ sang vermeil. On comprend ensuite que cette image photographique est posée dans une cuve de développement argentique, un dispositif qui connote l'apparition progressive d'une image destinée à se figer. Le sang ne provient pas

47. Émilie Bouvard, "Présence réelle et figurée du sang menstruel chez les artistes femmes : les pouvoirs médusants de l'auto-affirmation ». Communication réalisée dans le cadre de la Journée d'études "Les fluides corporels dans l'art contemporain " organisée à l'INHA, Paris, le 29 juin 2010, [http : //hicsa.univ-paris1.fr], dernière consultation le 24 septembre 2017.

48. Martine Beugnet, "EXPORTs Werden : Mann \& Frau \& Animal ", in Sylvia Szely (dir.), EXPORT Lexikon. Chronologie der bewegten Bilder bei VALIE EXPORT, Wien, Sonderzahl, 2007, p. 15.

49. Ibid, p. 16. 
de l'image du vagin, mais coule depuis l'avant-bras manifestement blessé d'un homme, tendu au-dessus de la cuve de développement.

La mise en scène de ce plan met en évidence la construction sociale de deux qualités différentes de sang dont la symbolique et la matérialité ne sont pas les mêmes : le sang qui coule depuis le bras de l'homme est indéniablement investi d'une dimension héroïque. En effet, si le sang en tant que sécrétion corporelle déversée doit toujours rester invisible pour être positivement identifié à la "sève de la vie" (celle qui circule dans les veines du Christ et qui se diffuse dans le monde à travers ses blessures), le sang ordinaire quand il est déversé volontairement, peut revêtir un caractère sacré ou héroïque dans le cadre d'une poétique du drame et de la douleur, comme celles que mettent en scène les religions, les nations en guerre ou encore le théâtre orgiaque des actionnistes viennois. Par opposition, le sang menstruel est considéré comme sale et impur, son écoulement involontaire comme une déperdition passive et incontrôlable. Dans Mann \& Frau \&t Animal, VALIE EXPORT nous montre l'“image" fixe d'un vagin, sans grande qualité haptique, qui contraste avec la main animée de l'homme qui, lui, procède au "don de sève" (ill. 3). Le sang menstruel s'oppose au sang jaillissant, l'“image" de la passivité féminine contraste avec l'héroïsme du sang versé. La sonorisation grotesque de cette séquence par des chants de baleines ne fait que souligner la dimension ironique de cette présentation où le corps féminin est dépossédé de la complexité de son image en même temps que de son flux menstruel.

Avec ses performances photographiées ou filmées qui exhibent le matériau des règles féminines comme flux corporel et scansion d'une temporalité féminine rétive aux récits surdéterminés, VALIE EXPORT a posé les premiers " cubes de construction média " d'une nouvelle perception des menstrues, bien avant l'essor de la question dans les débats féministes. L'apparition du sang menstruel est alors " un événement politique, lié au féminisme, mais aussi plastique " note Émilie Bouvard ${ }^{50}$. Car Menstruationsfilm et ses développements sont bien la réponse polémique à un contexte artistique précis, celui des actionnistes viennois que VALIE EXPORT fréquente durant les années $1960^{51}$. Ce qui fut pour elle une expérience de l'hétérodétermination séminale la mènera à se concentrer presque exclusivement sur l'objectivation de l'expérience féminine et la " matérialisation d'un devenir ", grâce notamment à la structure associative des techniques filmiques, tout en développant parallèlement une esthétique féministe qui cherche à échapper à une narrativité hétérodéterminée.

Aujourd'hui, les règles féminines restent toujours un terrain médiatique et artistique à conquérir. Dans le grand récit permissif de l'art contemporain, leur monstration semble en effet échapper à la logique constitutive de la provocation : en 2012, l'artiste portugaise Joana de Vasconcelos fut obligée de décrocher son grand lustre composé de tampons hygiéniques - pourtant immaculés -

50. Bouvard, " Présence réelle et figurée du sang menstruel " (en ligne).

51. Cf. Brigitta Burger-Utzer, « "In der Erweiterung liegt die Möglichkeit zur Veränderung”. Gespräch mit VALIE EXPORT », in Szely (dir.), EXPORT Lexikon, p. 210. 
à Versailles ${ }^{52}$; en 2015 les photographies - pourtant anodines - publiées par l'étudiante en art canadienne Rupi Kaur sur Instagram furent censurées par le service de partage de $\operatorname{photos}^{53}$. Mais le tabou des menstrues s'effrite, très souvent sur le mode de la dérision et par l'image qui montre leur réalité matérielle comme flux : en 2014, la photographe Marianne Rosenstihl exposait sa série intitulée The Curse [La malédiction] dans laquelle elle se moquait des récits ancestraux en présentant des femmes qui relèvent leur jupe pour "irriguer" un champ infertile ${ }^{54}$; en 2015 la musicienne indo-américaine Kiran Gandhi accomplissait le marathon de Londres tout en ayant ses règles et sans porter ni tampons ni serviettes périodiques afin de dénoncer la stigmatisation dont sont victimes les femmes dans le monde ${ }^{55}$; en 2016 , la stratégie publicitaire pour la marque Thinxs (des culottes chic cachant une nouvelle technologie très absorbante) ironisait sur le récit ancestral qui identifie les règles à un échec de la reproduction en figurant en gros plan un œuf de poule cassé dont la texture glaireuse s'écoule "vainement", simulant la matière du sang menstruel. Est-ce le signe que les femmes sont à la reconquête de leurs règles en déconstruisant les récits qui ont fortement contribué à les écarter de la cité?

\section{Annexes}

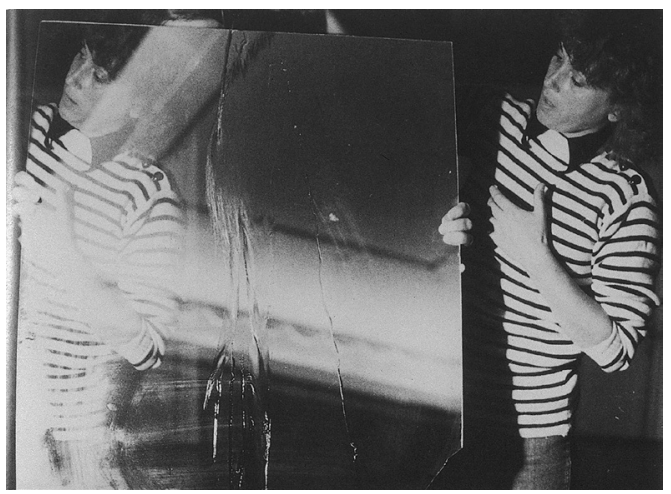

III. 1 : VALIE EXPORT, Abstract Film No. 1, 1967-1968, ㄷ VALIE EXPORT, Bildrecht Wien, 2018, courtesy VALIE EXPORT

52. Cf. Philippe Dagen, " Joana Vasconcelos, une artiste un peu trop libre pour la cour du RoiSoleil », Le Monde, 20 juin 2012.

53. Cf. Elise Thiébaud, Ceci est mon sang. Petite histoire des règles, de celles qui les ont et de ceux qui les font, Paris, La Découverte, 2017, p. 92-94.

54. "The Curse, la malédiction ", exposition à la galerie Petit Espace en décembre 2014, [https:// www.artlimited.net/agenda/marianne-rosenstiehl-the-curse-malediction-photographie-galeriepetit-espace-paris/fr/7582597], dernière consultation le 24 septembre 2017.

55. Thiébaud, Ceci est mon sang, p. 89-92. 


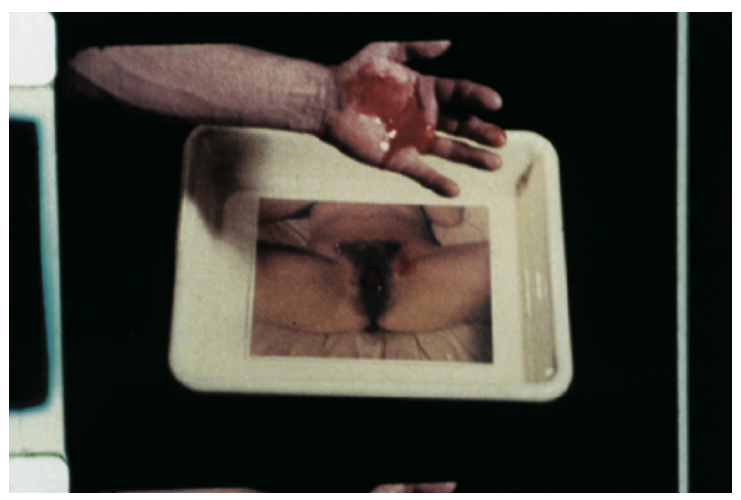

III. 2 : VALIE EXPORT, Menstruation, 1973, @ VALIE EXPORT, Bildrecht Wien, 2018, courtesy VALIE EXPORT

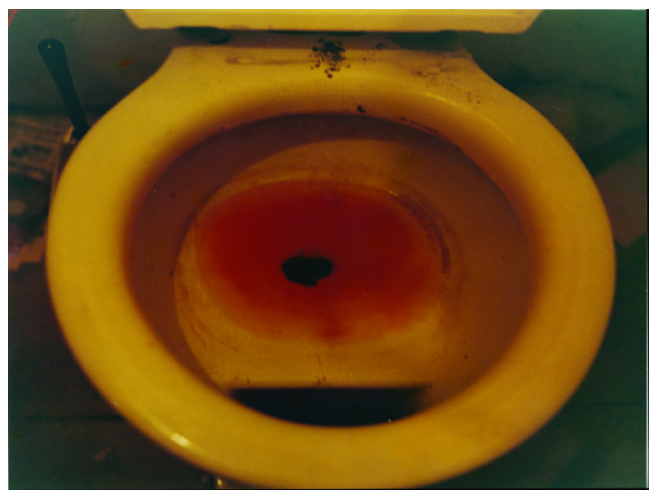

III. 3 : VALIE EXPORT, Mann \& Frau \& Animal, 1973, @ VALIE EXPORT, Bildrecht Wien, 2018, courtesy VALIE EXPORT 